\title{
Tourist' expectation and satisfaction towards pedestrian networks in the historical district of Kuala Lumpur, Malaysia
}

\begin{abstract}
Despite urban designers attempt to advocate walking in urban areas by upgrading the walking environment, there has been very little research done to corroborate this claim. More studies are required to investigate the connection between the walking environment and actual walking experience particularly in the context of Asian cities. This study discusses the effects of pedestrian accessibility, connectivity and continuity on tourists' walking experience based on their expectations and satisfaction of the pedestrian networks. The study focused on the historical district of Kuala Lumpur, Malaysia. Data were gathered from questionnaire survey conducted with 330 respondents, who were tourists walking in the area. The study demonstrated that tourists' expectations on the spatial characteristics of walkways in terms of accessibility, connectivity and continuity were greater than their satisfaction. The tourists' satisfaction relates strongly to diverse street usages and activities. The findings of the study will be a useful reference for planners and urban designers in providing walkable places to support urban tourism activities.
\end{abstract}

Keyword: Tourists' experience; Walkability; Expectation; Satisfaction; Accessibility 